\title{
A Polymorphic Ant Colony Algorithm (PACA) for the Selection of Optimized Band Selection of Hyperspectral Remote Sensing Image
}

\author{
Ding Xiaohui ${ }^{1,2}$, Zhang Shuqing ${ }^{1, a}$, Li Huapeng ${ }^{1}$ \\ 1 Northeast Institute of Geography and Agroecology, Chinese Academy of Sciences, Changchun 130102, China \\ 2 University of Chinese Academy of Sciences, Beijing 100049, China
}

Keywords: Hyperspectral, Dimensionality Reduction, Band Selection, Ant Colony Algorithm, Polymorphic Ant Colony Algorithm.

\begin{abstract}
With the definite labeled samples, it is difficult that avoid the curse of the dimensionality for the accurate and efficient classification of hyperspectral images. It is necessary that reduce the dimensionality of hyperspectral images. Therefore, Polymorphic Ant Colony Algorithm (PACA) based band selection algorithm (PACA-BS) for hyperspectral images is proposed in this paper. Compared with the common Ant Colony Algorithm (ACA) based band selection algorithm (ACA-BS), PACA-BS can significantly decrease the searching space and thus the time complexity. These algorithms are applied to select the bands of Hyperion and AVIRIS hyperspectral image according to the class separability criterion. Performance evaluation of algorithms is focused on the following aspects: computing time and overall classification accuracy. The results showed that the computing time of PACA-BS was markedly lower than ACA-BS. Furthermore, band sets of PACA-BS generate a higher overall classification accuracy. The PACA-BS is thus proved to be a promising and optimized method for band selection of hyperspectral image.
\end{abstract}

\section{Introduction}

Hyperspectral remote sensing, appeared in the 1980s, is one of the frontier domains of remote sensing technology ${ }^{[1]}$. Hyperspectral remote sensing imagery has lots of bands, high spectral resolution and continuous spectrum ${ }^{[2]}$, which contains abundant ground object information. However, it poses a great challenge for data storage, management, processing and analysis, due to its massive data volume and serious data redundancy ${ }^{[3]}$. To solve these problems, dimension reduction of the original hyperspectral image data is there necessary.

At present, methods for reducing data dimension can be broadly divided into two kinds, namely Feature Extraction and Feature Selection ${ }^{[4]}$. Feature extraction (e.g. principle component analysis (PCA)) is based on complex mathematical calculations, thus often time-consuming; moreover, the primitive physical significance of the image data is often lost in the process of data transformation, which is negative for the future usage of the post-processed data such as remote sensing information retrieval, visual interpretation, among others ${ }^{[5,6]}$. In contrary, feature selection (i.e. selecting the

\footnotetext{
${ }^{a}$ Corresponding author : zhangshuqing@neigae.ac.cn
} 
optimized remote sensing bands), capable of reserving the primitive physical significance, has received an increasing attention among remote sensing researchers. Yet, it is a typical NP-hard problem $^{[7]}$ to choose the optimized bands of hyperspectral imagery, which can only be effectively solved with a reasonable objective function and a proper searching algorithm. The determination of objective function is usually application-specific, i.e. relying on the objective of the application; whereas searching algorithms are hence considered as the key to the effective and efficient solution of optimal band selection.

There are two kinds of searching algorithms, namely global optimal searching algorithms and local optimal searching algorithms. Due to the high time complexity, the application of global optimal searching algorithms has been constrained. Developing proper optimal searching algorithms has thus been given first priority ${ }^{[8]}$. Ant Colony Algorithm (ACA) is one of the widely used local optimal algorithms because of its robustness and parallelism ${ }^{[9]}$. To reduce the searching space of the ants and improve the probability of achieving global optimum, polymorphic ant colony algorithm (PACA) was further presented ${ }^{[10]}$. In this study, an approach (denoted as PACA-BS for brief) to selecting the optimal bands of hyperspectral remote sensing image based on PACA was put forward. The performance of the proposed algorithm in terms of time complexity and classification that reflects the seperability of the selected optimal bands was comprehensively tested.

This paper is organized as follows: PACA was briefly introduced in section 2; the PACA-BS was presented in section 3; in section 4, the pheromone of hyperspectral dimension reduction of PACA-BS was tested and compared with traditional ACA using both Hyperion and AVIRIS imagery; a summary was made in the final section.

\section{Polymorphic ant colony algorithm (PACA)}

\subsection{Categories of ants}

Ants are divided into three categories, each of which is defined as follows:

Scouting Ant. Scouting ant simulates a scouting ant of natural ant colony that seeks food resources for searching ants and secretes scouting pheromone to guide them to the closest food resources ${ }^{[11]}$. Similarly, scouting ant in PACA can scout around to find the local optimal solution set and secrete scouting pheromone on the path.

Searching Ant. In a natural ant colony, searching ant can search along with the path to find and get the closest food resource ${ }^{[11]}$. In PACA, searching ant also finds the optimized solution set under the guidance of the scouting pheromone and secretes searching pheromone on the path to guide the following searching ants.

Working Ant. Natural working ant does not take part in the food searching process. It just gets the food from searching ants and feed its to the queen ant ${ }^{[11]}$. Working ants in PACA get the local optima first, and then evaluate them, according to the objective function, to achieve the global optimum.

\subsection{Mechanism of PACA}

In the nature, a social ant colony is composed by different functional ants, namely, scouting ants, searching ants, working ants and queen ant, etc. The scouting ants scout around for the near food resources and lay scouting pheromone on the path to guide the searching ants to find the nearest food source. For the searching ants, the behavior of a population of searching ant is more complex than a single searching ant, which can help the searching ants find the shortest path between the nest and the food source. While walking from the nest to the food resource, a searching ant excretes searching pheromone that forming pheromone trail on the path (trail-laying). Also, it can smell the pheromone and detect its concentration. In probability, the searching ant chooses the path marked by higher pheromone concentration (trail-following). In reference[12], an experiment was designed to explain 
how the collective behavior is generated from the single ant's trail-laying and trail-following behavior. It was also proved that the searching ant colony can find the shortest path by the trail-following behavior of the ant colony in reference[13]. Due to the faster searching pheromone accumulation on the shorter paths, the trail-following behavior employed by the searching ant colony can give rise to the emergency of the shortest path.

In PACA, laying $m_{l}$ scouting ants to $n$ instances $\left(m_{l}=n\right)$. The scouting ant scouts around instances which have short distance with the instance $C_{i}$ where the scouting ant is at. The value of is relevant to the number of instances, which is determined according to the statistic results (Tab.1) of maximal selectable instances ${ }^{[14]}$.

Table. 1 the statistic result of maximal selectable instances ${ }^{[14]}$

\section{Instances}

\begin{tabular}{ccc}
\hline 20 & 8 & 11 \\
100 & 9 & 55 \\
144 & 13 & 61 \\
1000 & 19 & 545 \\
\hline
\end{tabular}

The instances are recorded in the corresponding set, and the scouting ants excretes scouting pheromone on the paths. The concentration of the scouting pheromone is calculated as follows:

* MERGEFORMAT (1)

Where represents the length of the edge $(i, j)$ between th and th instance; denotes the minimal length of the path between th instance and one of the $n-1$ instances; is the MaxB set of $i$ th instances.

Thus, we can according Eq.(1) to initialize the searching pheromone in PACA as follows:

I* MERGEFORMAT (2)

Where is a user defined constant; represents the maximal distance between the th and the other $n-1$ instances.

Both ACA and PACA have the same status change strategy: the searching pheromone intensity of edge $(i, j)$ is higher, the probability that the searching ant select the path is more greater. The searching pheromone at $t$ time is updated as follows:

Where is the pheromone density, and is the total path length of the $k$ th ant; is the evaporation rate of the searching pheromone, which is a constant for all searching ants.

The probability of the th searching ant selecting th instance will be in line with the variation of searching pheromone between th instance and th instance, which is calculated as follows:

Where, represent information accumulated during the movement of ants and the different effects of factors in the path selection. . is the set of selectable instances.

As the description of working ant in section 2.1, the working ant evaluate the instances sets selected by the searching ants by equation (6). If the result of the objective function is improved, the corresponding instance set will be taken as the optimal solution set.

Where $m_{2}$ is the size of the searching ant colony.

\section{PACA-BS}

In order to overcome the shortcomings of ACA-BS ${ }^{[15]}$ which we designed for the optimized band selection of hyperspectral remote sensing imagery, we introduce the PACA into the field of optimal band selection of hyperspectral remote sensing imagery and design the PACA-BS. Due to the 
guidance of scouting ants, the searching spaces of searching ants are sharply decreased. Therefore, the running time of PACA-BS is decreased significantly compared with ACA-BS.

According to the literature [14], the should contains 19 bands which the correlation with the $i$ th band is lower than correlation coefficient threshold $T$ for the number of bands of the experimental data belongs to 144 to 1000 . Therefore, It means the probability that searching ants obtain the optimal band combination is far greater than $50 \%$.

The optimal band combination means there is a well separability between the bands which is measured by Bhattacharya distance $(B H)$. In PACA-BS, the objective function is, the reciprocal of $B H_{i j}$ between the th and the th band. and $B H_{i j}$ can be calculated as follows:

Where is the mean vector of class and is the corresponding covariance matrix.

We present the pseudo code of PACA-BS in Tab.2.

Table 2. the pseudo code of PACA-BS

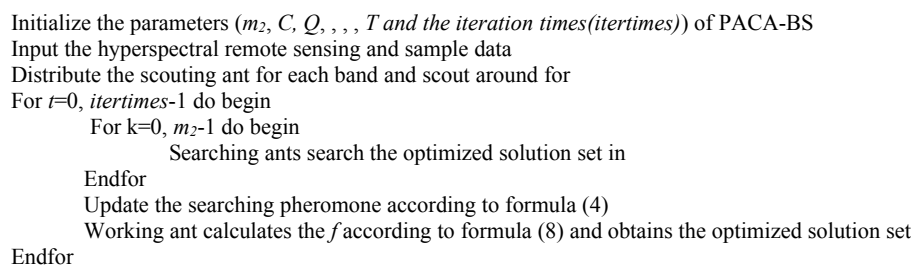

\section{Experiments}

Two hyperspectral images are used in this paper, i.e. Hyperion (Fig.1a) and AVIRIS (Fig.1b). After preprocessing, There were only 163 and 200 bands left respectively in the Hyperion and the AVIRIS image.

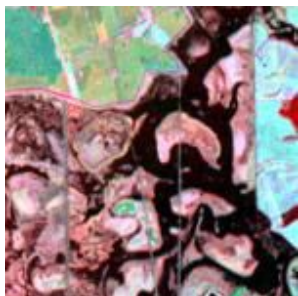

(a)

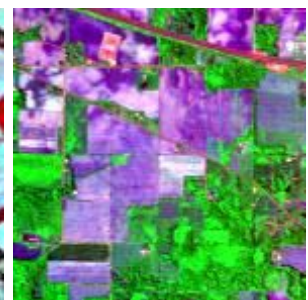

(b)

Figure 1. Original imagery of Hyperion and AVIRIS

In the following experiments, we adopted Maximal Likelihood Classifier (MLC) to classify the Hyperion and AVIRIS image into the 6 and 9 categories individually. We collected 976 samples for the classification of Hyperion image and 2138 samples for AVIRIS image. Then, we have selected about half of the samples randomly for classifier training. The rest samples were used to test the accuracy of MLC.

We compared the performances of ACA-BS and PACA-BS from the following aspects: computing time and overall accuracy. We set $m_{2}=50, Q=100, \alpha=1, \rho=0.5, T=0.9$ both in ACA-BS and PACA-BS and set $C=2$ in PACA-BS after many crossover experiments.

In the experiments, we adopted ACA-BS and PACA-BS to select 5, 10, 15, 20 and 25 bands respectively from Hyperion and AVIRIS image to combine into the band subset and test the algorithms for more than 10 times. We take the average of the running time and the overall accuracy for algorithms. Fig. 2 shows the result of algorithms. 


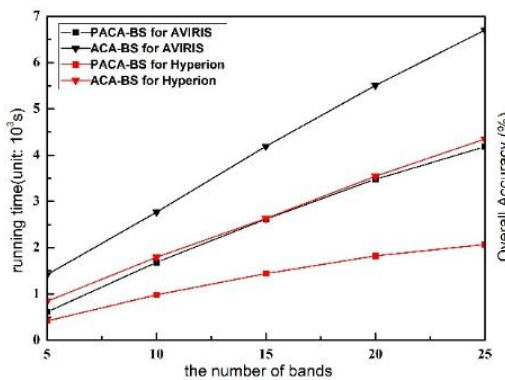

(a)

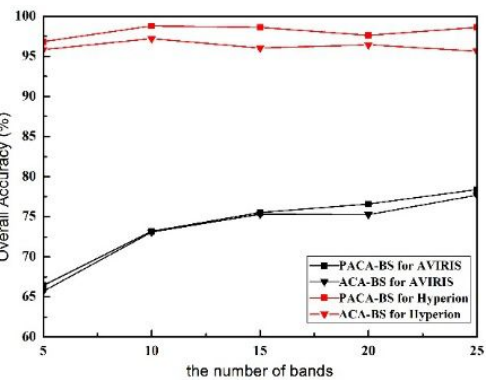

(b)

Figure 2. Contrastive results between the evaluation criteria of ACA-BS and PACA-BS

As can be seen from the Fig.2, the running time have been significantly decreased by PACA-BS for both AVIRIS and Hyperion imagery. It is the result of the markedly decreased searching space. The overall accuracy has been improved for the reason that PACA-BS can avoid run into local optimization and the separability have been improved. Tab.3 shows the overall accuracy and the kappa coefficient for Hyperion and AVIRIS imagery.

Table 3. the overall accuracy and the kappa coefficient for Hyperion and AVIRIS imagery

\begin{tabular}{|c|c|c|c|c|c|c|c|}
\hline & Nun & f bands & 5 & 10 & 15 & 20 & 25 \\
\hline \multirow{4}{*}{ AVIRIS } & \multirow[b]{2}{*}{ ACA-BS } & Accuracy (\%) & 65.7 & 73.06 & 75.31 & 75.26 & 77.70 \\
\hline & & Kappa & 0.62 & 0.69 & 0.72 & 0.74 & 0.74 \\
\hline & \multirow{2}{*}{ PACA-BS } & Accuracy (\%) & 66.43 & 73.19 & 75.52 & 76.2 & 78.39 \\
\hline & & Kappa & 0.63 & 0.70 & 0.72 & 0.74 & 0.75 \\
\hline \multirow{4}{*}{ Hyperion } & \multirow[b]{2}{*}{ ACA-BS } & Accuracy (\%) & 95.85 & 97.23 & 96.05 & 96.44 & 95.65 \\
\hline & & Kappa & 0.95 & 0.97 & 0.95 & 0.95 & 0.94 \\
\hline & \multirow[b]{2}{*}{ PACA-BS } & Accuracy (\%) & 96.84 & 98.81 & 98.62 & 97.63 & 98.62 \\
\hline & & Kappa & 0.96 & 0.99 & 0.98 & 0.97 & 0.98 \\
\hline
\end{tabular}

\section{Conclusion}

Due to the large searching space and local optimization, the performance of ACA-BS is not so satisfactory. In contrast, PACA-BS is more efficient and effective in the running time of algorithm and the classification accuracy of the band subset. It is the result of the interaction of different kinds of ants. The scouting ant which scout around for the local optimization, significantly decrease the searching space. Therefore, the probability that searching ant get the global optimization is improved. The PACA-BS is thus proved to be a promising and optimized method for band selection of hyperspectral image. 


\section{References}

[1] Tong Qingxi, Zhang Bing, Zheng Lanfen. Hyperspectral Remote Sensing[M].Beijing:Higher Education Press, 2006.

[2] Zhang Liangpei, Zhang Lifu. Hyperspectral Remote Sensing[M]. Wuhan: Wuhan University Press, 2005.

[3] Chengfu Huo, Rong Zhang, Dong Yin. Compression technique for compressed sensing hyperspectral images[J]. International Journal of Remote Sensing, 2012, 33(5): 1586-1604.

[4] Kittler J. Feature selection and extraction[J]. Handbook of pattern recognition and image processing, 1986: 59-83.

[5] XinQin, Nian Yongjian, Li Xiu, et al. Dimensionality reduction for hyperspectral imagery based on fastica [J]. JOURNAL OF ELECTRONICS (CHINA), 2009, 26(6): 831-835.

[6] Mei Anxin, Peng Wanglu, Qin Qiming. An introduction to Remote Sensing[M]. Beijing: Higher Education Press, 2001.

[7] Liang Feng, Ah-Hwee Tan, Meng-Hiot Lim, etc. Band selection for hyperspectral images using probabilistic memetic algorithm[J]. Soft Computing, 2014, 18(1): 1-9.

[8] Markand P.Oza. Selection of band combination for irs data[J]. Journal of the Indian Society of Remote Sensing, 1989, 17(2): 23-26.

[9] Huang Zhixiong, Zhang Dengke, Li Qunhui. Ant Colony Algorithm and Summary of Its Improved Algorithm[J]. Computing Technology and Automation, 2006, 25(3): 35-38.

[10] Xu Jingming, Cao Xianbin, Wang Xufa. Polymorphic Ant Colony Algorithm[J]. Journal of university science and technology of China, 2005, 35(1): 59-65.

[11] Yang Pei. Ant colony society biology and diversity[J]. Entomological knowledge, 1999, 36(4): 243-247.

[12] Deneubourg J L, Aron S, Goss S, et al. The self-organizing exploratory pattern of the argentine ant[J]. Journal of insect behavior, 1990, 3(2): 159-168.

[13] Dorigo M, Caro G D, Gambardella L M. Ant algorithms for discrete optimization[J]. Artificial life, 1999, 5(2): 137-172.

[14] Quan Huiwen, Wen Gaojin. Subspacegeneticalgorithm forTSP[J]. MATHEMATICAL THEORY AND APPLICATIONS, 2002, 22(1): 36-39.

[15] Zhou S, Zhang J, Su B. Feature selection and classification based on ant colony algorithm for hyperspectral remote sensing images[C]//Image and Signal Processing, 2009. CISP'09. 2nd International Congress on. IEEE, 2009: 1-4. 